\title{
Remoção de fósforo em sistema de tratamento de esgoto doméstico, por escoamento superficial ${ }^{1}$
}

\author{
Ana P. S. Loures², Antônio A. Soares², Antônio T. de Matos², Paulo R. Cecon² \& Odilon G. Pereira ${ }^{2}$
}

\begin{abstract}
RESUMO
Com o objetivo de avaliar a remoção e a concentração de fósforo $(\mathrm{P})$ no esgoto doméstico, conduziram-se ensaios de campo, utilizando-se o método de escoamento superficial, sob diferentes taxas de aplicação: 0,24; 0,36 e 0,48 $\mathrm{m}^{3} \mathrm{~h}^{-1} \mathrm{~m}^{-1}$. Nas amostras de esgoto verificou-se que a taxa de aplicação $0,24 \mathrm{~m}^{3} \mathrm{~h}^{-1} \mathrm{~m}^{-1}$ proporcionou as maiores taxas de remoção de P: 47,46\% para P total; 47,45\% para P total dissolvido; 42,60\% para P inorgânico dissolvido e $85,12 \%$ para P orgânico dissolvido; entretanto, para $P$ total no resíduo, a taxa de aplicação $0,36 \mathrm{~m}^{3} \mathrm{~h}^{-1} \mathrm{~m}^{-1}$ resultou na maior eficiência de remoção de P: 55,65\%. Para avaliar o solo ao longo do seu perfil coletaram-se amostras antes e depois da aplicação do esgoto, em duas camadas: de 0 a $10 \mathrm{~cm}\left(\mathrm{C}_{1}\right)$ e de 50 a $60 \mathrm{~cm}\left(\mathrm{C}_{2}\right)$. Para as taxas de aplicação 0,24 e 0,48 $\mathrm{m}^{3} \mathrm{~h}^{-1} \mathrm{~m}^{-1}$ verificou-se, em ambas as camadas, diminuição no conteúdo de P disponível: para $0,24 \mathrm{~m}^{3} \mathrm{~h}^{-1} \mathrm{~m}^{-1}, 21 \%$ em $\mathrm{C}_{1}$ e $63 \%$ em $\mathrm{C}_{2}$; e, para $0,48 \mathrm{~m}^{3} \mathrm{~h}^{-1} \mathrm{~m}^{-1}, 70 \%$ em $\mathrm{C}_{1}$ e $57 \%$ em $\mathrm{C}_{2}$; porém, para a taxa $0,36 \mathrm{~m}^{3} \mathrm{~h}^{-1} \mathrm{~m}^{-1}$ constatou-se, em ambas as camadas, aumento na referida variável: $8 \%$ em $\mathrm{C}_{1}$ e $59 \%$ em $\mathrm{C}_{2}$. Para avaliar a capacidade da forrageira em remover $\mathrm{P}$ efetuaram-se 3 cortes em área de $1 \mathrm{~m}^{2}$, ressaltando-se que a taxa $0,36 \mathrm{~m}^{3} \mathrm{~h}^{-1} \mathrm{~m}^{-1}$ proporcionou o maior valor de remoção de P pelo capim-coastcross: $15,78 \mathrm{~kg} \mathrm{ha}^{-1}$ de P em um total de 137 dias de avaliação.
\end{abstract}

Palavras-chave: água residuária, capim-coastcross, remoção de fósforo, tratamento no solo

\section{Phosphorus removal in overland flow system for domestic wastewater treatment}

\begin{abstract}
In order to evaluate the overland flow treatment efficiency for removing phosphorus $(\mathrm{P})$ at different application rates: $0.24,0.36$ and $0.48 \mathrm{~m}^{3} \mathrm{~h}^{-1} \mathrm{~m}^{-1}$ of domestic wastewater, in flow slopes with a down-slope grade of $2 \%$, an experiment was developed in the experimental area of the Agricultural Engineering Department of the Federal University of Viçosa. For the biomass production the 'coastcross' bermudagrass Cynodon dactylon was used. The highest removal rates for samples collected at $8 \mathrm{~m}$ from the beginning of the borders occurred for the application rate of $0.24 \mathrm{~m}^{3} \mathrm{~h}^{-1} \mathrm{~m}^{-1}: 47.46 \%$ for total-P; $47.45 \%$ for total dissolved-P; $42.60 \%$ for inorganic dissolved-P; and $85.12 \%$ for organic dissolved-P. However, for total suspended-P, the highest removal rate (55.65\%) occurred for the application rate of $0.36 \mathrm{~m}^{3} \mathrm{~h}^{-1} \mathrm{~m}^{-1}$. Soils samples were collected in two layers at $1 \mathrm{~m}$ from upper end: $L_{1}(0$ to $10 \mathrm{~cm})$ and $L_{2}(50$ to $60 \mathrm{~cm})$. A decrease in $P$ for both layers for the treatments with 0.24 and $0.48 \mathrm{~m}^{3} \mathrm{~h}^{-1} \mathrm{~m}^{-1}$ of application rate was detected. In an opposite way, the amount of $\mathrm{P}$ for the treatment with $0.36 \mathrm{~m}^{3} \mathrm{~h}^{-1} \mathrm{~m}^{-1}$ increased. The treatment with $0.36 \mathrm{~m}^{3} \mathrm{~h}^{-1} \mathrm{~m}^{-1}$ of application rate, in 137 days, presented the highest values of $\mathrm{P}$ removals by the 'coastcross' bermudagrass.
\end{abstract}

Key words: wastewater, coastcross bermudagrass, phosphorus removal, treatment on soil

1 Parte da Dissertação de Mestrado do primeiro autor, apresentada à Universidade Federal de Viçosa

${ }^{2}$ UFV. Av. PH. Rolfs s/n, CEP 36570-000, Viçosa, MG. Fone (31) 3899-2730. Fax: (31) 3899-2735. E-mail: aploures@vicosa.ufv.br; aasoares@ufv.br; atmatos@ufv.br; cecon@dpi.ufv.br; odilon@ufv.br 


\section{INTRODUÇÃO}

O fato do esgoto doméstico constituir rica fonte de matéria orgânica e nutrientes minerais fez com que deixasse de ser visto apenas como despejo residual e passasse a ser considerado um recurso hídrico aproveitável (Metcalf \& Eddy, 2003), desde que atenda às exigências sanitárias. Assim, as técnicas de tratamento visando ao aproveitamento das águas residuárias domésticas ganharam impulso, principalmente aquelas que aumentam a produção de biomassa vegetal, a ser utilizada diretamente na alimentação humana ou animal. Uma das técnicas que têm recebido especial atenção é a de tratamento de esgotos pelo método de escoamento superficial.

No esgoto bruto, há quantidades substanciais de fósforo, nas formas: orgânica; inorgânica complexa (polifosfatos), como aquelas utilizadas em detergentes; e ortofosfato inorgânico solúvel, este como produto final no ciclo do fósforo, a forma mais prontamente disponível para uso biológico (Black, 1980). Durante o processo de tratamento biológico, os compostos orgânicos são degradados, podendo disponibilizar ortofosfatos solúveis e polifosfatos que, quando hidrolisados, podem ser convertidos em ortofosfatos. Em um efluente orgânico bem-estabilizado submetido a tratamento secundário, o ortofosfato é a forma predominante do fósforo, que pode ser removido por processos de precipitação química ou absorvido por plantas e microrganismos.

Uma preocupação crescente em relação ao fósforo está relacionada à eutrofização das águas. Esteves (1988) definiu a eutrofização como o aumento da concentração de nutrientes, especialmente $\mathrm{P}$ e $\mathrm{N}$, nos ecossistemas aquáticos, que tem como conseqüência o crescimento descontrolado de algas e de plantas aquáticas. A eutrofização pode ser natural ou artificial, sendo esta induzida pelas atividades humanas e tendo os nutrientes diferentes origens, como: efluentes domésticos e industriais e/ou atividades agrícolas, entre outras. A eutrofização artificial é um processo dinâmico, no qual ocorrem profundas modificações qualitativas e quantitativas nas comunidades aquáticas, nas condições físicas e químicas do meio e no nível de produção do sistema.

Quando excessivo em ambientes aquáticos, o fósforo tem sido considerado responsável por problemas causados pela eutrofização (Novais \& Smyth, 1999). O transporte de fósforo de solos para ambientes aquáticos, via escoamento superficial e sub-superficial, pode criar condições nutricionais favoráveis ao crescimento acima do normal de fito e zooplâncton, além de plantas aquáticas superiores. Este crescimento exagerado de biomassa causa aumento na demanda biológica de oxigênio, alteração de $\mathrm{pH}$, turbidez da água, liberação de toxinas, que podem causar morte de peixes, de animais etc.

Heckrath et al. (1995), citados por Novais \& Smyth (1999), registraram que a concentração crítica inicial para o estabelecimento de efeitos de eutrofização em lagos pode ser tão baixa como $0,02 \mathrm{mg} \mathrm{L}^{-1}$ de P. A Resolução CONAMA $\mathrm{n}^{\circ} 357$, de 17 de março de 2005, estabelece o limite de 0,020 a $0,15 \mathrm{mg} \mathrm{L}^{-1}$ de $\mathrm{P}$ para fósforo total, de acordo com a classificação das águas doces em classes 1, 2 e 3, estabelecendo que, não havendo metas obrigatórias progressivas, intermediárias e final para este parâmetro no efluente a ser lançado, direta ou indiretamente, nos corpos d'água, o padrão de qualidade a ser obedecido será aquele que consta na classe na qual o corpo receptor estiver enquadrado (Brasil, 2005).

Na disposição dos esgotos no solo por escoamento superficial, a remoção do fósforo dá-se: por precipitação química com alumínio e ferro e na forma de fosfato de cálcio, $\mathrm{Ca}_{3}\left(\mathrm{PO}_{4}\right)_{2}$, por adsorção no complexo de troca e na superfície das argilas, e por absorção pela vegetação em crescimento nas rampas de tratamento (Paganini, 1997). Para que este último fator de remoção do fósforo possa ser potencializado, recomenda-se que a freqüência de cortes e a retirada da biomassa sejam as mais numerosas possíveis, minimizando sua concentração no solo da área de tratamento e, assim, possibilitando maior sustentabilidade ao sistema. O grau de remoção do fósforo neste sistema de tratamento depende do grau de contato entre o esgoto e a matriz do solo. Quando estão presentes solos rasos com baixa permeabilidade, como é o caso da maioria dos sistemas de escoamento superficial, muito da água residuária aplicada escoa sobre a superfície com pequeno contato com a matriz do solo e com os sítios de adsorção de fósforo. A remoção de fósforo nos sistemas de escoamento superficial varia de 40 a 60\% (USEPA, 1981), sendo o valor médio de remoção de aproximadamente 50\% (Coraucci Filho, 1991; Campos, 1999).

Segundo Abernathy et al. (1985), para o dimensionamento de sistemas de tratamento de águas residuárias por escoamento superficial, o modelo mais comumente encontrado na literatura utiliza o comprimento da rampa de escoamento como única variável independente. Espera-se que o tempo de contato e o desempenho do sistema de tratamento estejam diretamente relacionados e que aumentos no tempo de contato produzam melhor desempenho do sistema de tratamento; no entanto, uma relação para simular o tempo de detenção do líquido em uma rampa de escoamento superficial, baseada em suas características físicas e operacionais, tais como declividade, distância de escoamento e taxa de aplicação, não está disponível, sendo o comprimento da rampa usado como referencial para o tempo de contato entre a água residuária e o filme biológico.

Smith \& Schroeder (1985), baseados em resultados de estudo piloto em Davis, Califórnia, e utilizando esgoto doméstico, concluíram que a remoção do material orgânico, expresso como demanda bioquímica de oxigênio $\left(\mathrm{DBO}_{5}\right)$, do efluente primário (efluente bruto após etapa de decantação) poderia ser descrita por um modelo com dois estádios, em função apenas do comprimento da rampa e da taxa de aplicação. De acordo com esses autores, a provável razão para a existência de dois estádios decorria da remoção de maior quantidade de matéria orgânica (MO) sedimentável nos primeiros metros de rampa, permanecendo grande parte do material orgânico coloidal e solúvel para ser removido em menor taxa, por degradação bacteriana, adsorção na matriz do solo e absorção pela biomassa no comprimento restante da rampa.

Os propósitos deste trabalho foram: i) avaliar a capacidade de rampas de escoamento superficial como método de 
tratamento na remoção de diferentes formas de fósforo presentes em esgoto doméstico, após tratamento preliminar; ii) obter as equações de remoção de fósforo em função do comprimento de rampa; e iii) avaliar possíveis alterações em características químicas do solo em decorrência da aplicação de água residuária em três diferentes taxas no solo.

\section{MATERIAL E MÉTODOS}

O trabalho de campo foi realizado no período de julho a novembro de 2001, utilizando-se o método de escoamento superficial, nas dependências da EETE (Estação Experimental de Tratamento de Esgoto) do Departamento de Engenharia Agrícola, da Universidade Federal de Viçosa (UFV), Viçosa, MG (coordenadas: $20^{\circ} 45^{\prime} 14^{\prime \prime} \mathrm{S}$; 42 ${ }^{\circ} 52^{\prime}$ 53” W; altitude média de $648 \mathrm{~m}$ ).

O esgoto doméstico utilizado no experimento provinha do conjunto residencial Condomínio Bosque do Acamari, constituído por 136 residências e situado próximo à UFV.

Para condução do esgoto até o local do experimento, foi construída, na área da Dendrologia do Departamento de Engenharia Florestal, uma Estação Elevatória de Esgoto (EEE), dotada de duas bombas submersíveis de 1,0 cv, sendo uma reserva, com vazão de $1,86 \mathrm{~L} \mathrm{~s}^{-1}$ e altura manométrica de 14 mca, para recalcar o esgoto até a EETE, por meio de $430 \mathrm{~m}$ de emissário em PVC e diâmetro de $75 \mathrm{~mm}$.

A EETE consistiu de tratamentos preliminar e secundário (Figura 1). O tratamento preliminar foi formado por sistema de gradeamento, desarenador, canal para realização de medição de vazão em vertedouro de seção triangular e tanque de equalização de $1 \mathrm{~m}^{3}$, sendo o esgoto misturado por hélice acionada a motor de $2,0 \mathrm{cv}$ e $3.500 \mathrm{rpm}$. O tratamento secundário ocorreu por disposição no solo em uma

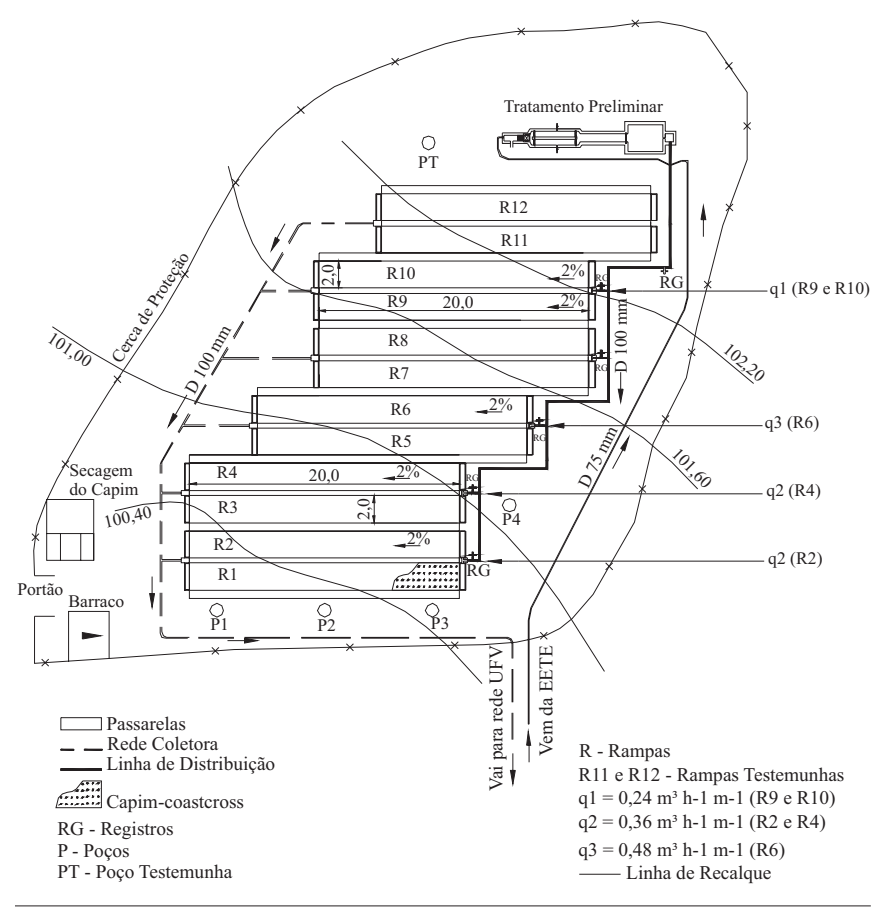

Figura 1. Croqui da estação experimental de tratamento de esgoto área dividida em seis planos de sistematização, cada um com duas rampas de $2 \mathrm{~m}$ de largura, $20 \mathrm{~m}$ de comprimento e declividade de $2 \%$, cultivadas com capim-coastcross (Cynodon dactylon).

Utilizaram-se três taxas de aplicação de esgoto, após tratamento preliminar: 0,$24 ; 0,36$ e $0,48 \mathrm{~m}^{3} \mathrm{~h}^{-1} \mathrm{~m}^{-1}$. As taxas de $0,24\left(\mathrm{q}_{1}\right)$ e $0,36 \mathrm{~m}^{3} \mathrm{~h}^{-1} \mathrm{~m}^{-1}\left(\mathrm{q}_{2}\right)$ foram aplicadas em rampas de tratamento duplicadas, $R_{9}$ e $R_{10}$ e $R_{2}$ e $R_{4}$, respectivamente, enquanto a taxa de $0,48 \mathrm{~m}^{3} \mathrm{~h}^{-1} \mathrm{~m}^{-1}\left(\mathrm{q}_{3}\right)$ foi aplicada em apenas uma rampa de tratamento $\left(R_{6}\right)$. O esgoto foi distribuído com uso de tubos em PVC, diâmetro de $100 \mathrm{~mm}$ e extensão de $50 \mathrm{~m}$. O controle da vazão foi feito, para o ajuste preliminar, com o emprego de registros de gaveta de $100 \mathrm{~mm}$, instalados na entrada de cada plano de sistematização e, para o ajuste fino, com torneiras instaladas na entrada de cada rampa. Construíram-se canaletas de concreto no início de cada rampa para uniformizar a aplicação do esgoto desarenado e, no final, para recolhimento do efluente final e sua condução por meio de tubos em PVC, diâmetro de $100 \mathrm{~mm}$, para a rede de esgoto da UFV.

O funcionamento do sistema de tratamento consistiu na aplicação de esgoto pelo período de $8 \mathrm{~h} \mathrm{dia}^{-1}$, de segunda a sexta-feira, e descanso aos sábados, domingos e feriados; portanto, com freqüência de aplicação de 5 dias/semana.

A Tabela 1 apresenta dados meteorológicos diários, obtidos durante o período de monitoramento da EETE.

Tabela 1. Dados meteorológicos dos dias de coleta de amostras nas rampas de escoamento superficial em experimento realizado na Universidade Federal de Viçosa, MG

\begin{tabular}{|c|c|c|c|c|c|c|}
\hline $\begin{array}{c}\text { Data } \\
\text { de Coleta }\end{array}$ & $\begin{array}{c}\mathrm{T} \\
\left({ }^{\circ} \mathrm{C}\right)\end{array}$ & $\begin{array}{l}\text { UR } \\
\text { (\%) }\end{array}$ & $\begin{array}{c}\text { I } \\
\text { (h) }\end{array}$ & $\begin{array}{c}P \\
(\mathrm{mb})\end{array}$ & $\begin{array}{c}\mathrm{Pe} \\
(\mathrm{mm})\end{array}$ & $\begin{array}{c}\text { Ev } \\
(\mathrm{mm})\end{array}$ \\
\hline 04/07/01 & 15,3 & 75 & 9,9 & 939,3 & 0,0 & 2,4 \\
\hline $17 / 07 / 01$ & 14,2 & 80 & 10,5 & 940,2 & 0,0 & 3,0 \\
\hline $31 / 07 / 01$ & 16,5 & 72 & 8,5 & 944,0 & 0,0 & 3,7 \\
\hline $14 / 08 / 01$ & 17,3 & 74 & 10,7 & 943,3 & 0,0 & 4,0 \\
\hline $29 / 08 / 01$ & 19,7 & 85 & 7,5 & 925,8 & 0,0 & 3,2 \\
\hline $12 / 09 / 01$ & 16,3 & 93 & 0,0 & 938,9 & 0,0 & 5,2 \\
\hline $25 / 09 / 01$ & 20,9 & 69 & 4,8 & 936,7 & 0,0 & 3,7 \\
\hline $23 / 10 / 01$ & 21,7 & 69 & 12,3 & 935,2 & 6,5 & 2,2 \\
\hline $07 / 11 / 01$ & 23,4 & 75 & 9,2 & 937,5 & 1,5 & 2,4 \\
\hline $21 / 11 / 01$ & 20,6 & 82 & 2,1 & 937,8 & 10,5 & 1,0 \\
\hline
\end{tabular}

P - Pressão atmosférica; Ev - Evaporaçãa total

Amostras líquidas do esgoto foram coletadas com seringas descartáveis de $20 \mathrm{~mL}$ na entrada do tratamento secundário, ou seja, no início e ao longo de cada rampa, nas distâncias de 2, 4, 6, 8 e 10 m. Em cada dia de coleta eram feitas três coletas de $100 \mathrm{~mL}$ em cada ponto gerando-se, assim, amostras compostas. Em geral, as coletas foram realizadas quinzenalmente, nas terças ou quartas-feiras, no intervalo das 9 às $15 \mathrm{~h}$, correspondentes aos dias e horários de maior concentração de carga orgânica no esgoto doméstico (Fonseca, 2000).

As análises do esgoto foram efetuadas no Laboratório de Qualidade da Água do Departamento de Engenharia Agrícola (DEA) da UFV, sendo o fósforo total (Pt), o fósforo 
total dissolvido (Ptd) e o fósforo inorgânico dissolvido (Pid) analisados pelo método 4500-P B e E, descritos no Standard Methods (APHA; AWWA; WEF, 1992). Para fósforo total no resíduo (Ptr), referente aos sólidos suspensos e coloidais presentes no esgoto doméstico, obtiveram-se os resultados por diferença entre Pt e Ptd e, para fósforo orgânico dissolvido (Pod), por diferença entre Ptd e Pid. As análises foram realizadas em duplicata, para cada ponto.

Adotou-se o modelo de aproximação da distância ao longo do comprimento para descrever a remoção dos nutrientes. As concentrações em cada ponto de amostragem (C), em relação à concentração afluente $\left(\mathrm{C}_{0}\right)$, foram determinadas para cada evento de amostragem $\left(\mathrm{C} / \mathrm{C}_{0}\right)$. Esses valores foram utilizados para ajustar os parâmetros dos modelos linear e exponencial.

O estádio 1 do modelo é aplicado aos primeiros metros do comprimento da rampa, nos quais a taxa de remoção orgânica é maior, e o estádio 2 do modelo, para o restante da rampa. Assim:

$$
\begin{array}{ll}
\text { Estádio } 1(\mathrm{x}<9 \mathrm{~m}): & \frac{\mathrm{C}_{\mathrm{x}}}{\mathrm{C}_{0}}=\mathrm{A} \exp (-\mathrm{K} \mathrm{x}) \\
\text { Estádio } 2(\mathrm{x}>9 \mathrm{~m}): & \frac{\mathrm{C}_{\mathrm{x}}}{\mathrm{C}_{0}}=\mathrm{A}^{\prime} \exp \left(-\mathrm{K}^{\prime} \mathrm{x}\right)
\end{array}
$$

em que:

$\mathrm{C}_{\mathrm{x}}$ - concentração de $\mathrm{P}$ na distância $\mathrm{x}$ na rampa de escoamento, mg L $\mathrm{m}^{-1}$

$\mathrm{C}_{0}$ - concentração de $\mathrm{P}$ no início da rampa de escoamento, mg $\mathrm{L}^{-1}$

A, A' - coeficientes de ajuste determinados empiricamente

K, K' - coeficientes de ajuste determinados empiricamente

x - distância na rampa de escoamento, m

Smith \& Schroeder (1985) concluíram, em estudo piloto, que os coeficientes $K$ e $K$ ’ nas equações 1 e 2 variaram como função da taxa de aplicação q, de acordo com as seguintes equações:

$$
\begin{array}{ll}
\text { Estádio } 1(\mathrm{x}<9 \mathrm{~m}): & \mathrm{K}=\frac{\mathrm{k}}{\mathrm{q}^{\mathrm{n}}} \\
\text { Estádio } 2(\mathrm{x}>9 \mathrm{~m}): & \mathrm{K}^{\prime}=\frac{\mathrm{k}^{\prime}}{\mathrm{q}^{\mathrm{n}^{\prime}}}
\end{array}
$$

em que:

$$
\begin{aligned}
& \text { k, k' - coeficientes determinados empiricamente, } \mathrm{m} \mathrm{h}^{-1} \\
& \text { q - taxa de aplicação, } \mathrm{m}^{3} \mathrm{~h}^{-1} \mathrm{~m}^{-1} \text { de largura da rampa } \\
& \text { n, n' - coeficientes determinados empiricamente }
\end{aligned}
$$

Para o modelo exponencial os valores de $\mathrm{k}$ e $\mathrm{n}$ foram determinados por meio de regressão linear de $\ln \mathrm{K}$ versus ln q.

O solo da área é classificado como Argissolo VermelhoAmarelo de textura argilosa (EMBRAPA, 1999). Para avaliação das alterações em relação ao conteúdo de fósforo ao longo do seu perfil, coletaram-se amostras por ocasião do início e do término do experimento, em duas camadas: de 0 a 10 e de 50 a 60 cm, na distância de $1 \mathrm{~m}$ do início da rampa, com o uso de trado tipo "Uland".

As análises de solo foram realizadas no Laboratório de Análise de Rotina de Solos do Departamento de Solos (DPS) da UFV, sendo: o P-disponível, pelo extrator Mehlich 1, analisado pelo método colorimétrico; a matéria orgânica (MO), pelo método Walkley-Black; o pH em água relação 1:2,5; e a acidez potencial $(\mathrm{H}+\mathrm{Al})$, pelo extrator acetato de cálcio 0,5 mol L-1 a pH 7,0 (Alvarez Venegas et al., 1999; EMBRAPA, 1997; Silva, 1999).

Para avaliar a capacidade da forrageira em remover fósforo, efetuaram-se três cortes na distância de $1 \mathrm{~m}$ do início da rampa, em área de $1 \mathrm{~m}^{2}$, com intervalo de aproximadamente 45 dias entre os cortes, totalizando um período de avaliação de 137 dias. As amostras da forrageira para análise foram coletadas com uso de cutelo, na distância de $10 \mathrm{~cm}$ do solo, e o capim restante foi cortado com uso de roçadeira costal e retirado manualmente das rampas de escoamento superficial.

As amostras da forrageira foram pesadas no próprio local do experimento, para determinação da produção de matéria verde; em seguida, transportou-se o capim para o Laboratório de Água e Solo do Departamento de Engenharia Agrícola (DEA), onde foi separado em sub-amostras as quais foram pesadas e secadas em estufa com circulação forçada de ar, sob temperatura de $65^{\circ} \mathrm{C}$, pelo período de $72 \mathrm{~h}$; depois, foram encaminhadas ao Laboratório de Nutrição Animal do Departamento de Zootecnia (DZO) da UFV, onde foram moídas em moinho tipo Willey para análise posterior de P. Para correção do teor de matéria seca (MS), sub-amostras foram secadas em estufa com convecção forçada de ar, sob temperatura de $108{ }^{\circ} \mathrm{C}$. Para a análise de fósforo, as amostras foram mineralizadas por digestão nítrico-perclórico e as concentrações, determinadas por colorimetria, segundo Silva \& Queiroz (2002).

Para efeito de análise estatística, considerou-se o experimento montado no delineamento inteiramente casualizado, com tratamentos (distância) e repetições diferentes para cada rampa. Os dados foram analisados para cada rampa, por meio de análise de regressão. As análises estatísticas foram realizadas utilizando-se o software Sistemas para Análises Estatísticas (SAEG) versão 8.0, desenvolvido na UFV.

\section{RESULTADOS E DISCUSSÃO}

\section{Remoção de fósforo na água residuária em escoamento nas rampas de tratamento}

A indisponibilidade de dados a partir dos $10 \mathrm{~m}$ de comprimento das rampas de escoamento superficial se deve à operação do sistema pelo período de apenas cinco meses, quando a literatura registra um período inicial de adaptação de 3 a 4 meses (USEPA, 1981), o que pode ser considerado reduzido para completa formação do filme biológico; além disso, as taxas de aplicação podem ter sido mais baixas que 
o necessário para promover a saturação da camada superficial do solo, ao longo do comprimento total da rampa.

Foi construído um dreno aos $10 \mathrm{~m}$ de comprimento da rampa durante a implantação do sistema de tratamento em período anterior ao da condução deste experimento. Mesmo depois de executadas obras para lacrá-lo devidamente, o meio da rampa permaneceu como um ponto crítico, em termos de permeabilidade, durante a operação do sistema.

Outro aspecto que dificultou o escoamento superficial foi a existência de caminhos preferenciais de deslocamento da água residuária devido à formação de fissuras no solo, durante o período em que o sistema de tratamento permaneceu em obras de manutenção, e à possibilidade de ocorrência de escoamento subsuperficial pelo fato das rampas de tratamento terem sido construídas perpendicularmente às curvas de nível.

Percebeu-se tendência da eficiência de remoção de fósforo aumentar com o comprimento da rampa de escoamento, Tabela 2, porém verificou-se, algumas vezes, aumento e não decréscimo do elemento (remoção negativa) em razão da oscilação da concentração do afluente nos diferentes dias de coleta e ao fato do esgoto, ao escoar sobre a superfície do solo, arrastar partículas de argila, material orgânico prove- niente do filme biológico, o qual se desprende da superfície solo-planta, e material vegetal proveniente do corte do capim que, devido às pequenas dimensões, não foi removido da rampa, onde sofreu decomposição. A camada de lodo que se acumulou no início da rampa também deve ter contribuído para o aumento das concentrações médias de fósforo, principalmente aos $2 \mathrm{~m}$ do início da rampa.

Considerando-se a eficiência de remoção de $47,46 \%$ para Pt, percebe-se que este valor está dentro do intervalo de 40 a 60\%, registrado por USEPA (1981), e próximo ao valor médio de 50\% aproximadamente, citado por Coraucci Filho (1991) e Campos (1999). É importante ressaltar que as eficiências encontradas neste trabalho se referem à fase inicial do tratamento, correspondente aos primeiros metros da rampa e não à eficiência total do sistema de tratamento.

Para a maioria das variáveis avaliadas, a taxa de aplicação $0,24 \mathrm{~m}^{3} \mathrm{~h}^{-1} \mathrm{~m}^{-1}$ alcançou as maiores taxas de remoção e a taxa $0,48 \mathrm{~m}^{3} \mathrm{~h}^{-1} \mathrm{~m}^{-1}$, as menores, o que condiz com os resultados encontrados por outros autores. Coraucci Filho (1991) registrou, no primeiro ano de seu experimento, a remoção média no Pt de $80 \%$ para a taxa de aplicação $0,15 \mathrm{~m}^{3} \mathrm{~h}^{-1} \mathrm{~m}^{-1}$ e de $55 \%$ para a taxa $0,30 \mathrm{~m}^{3} \mathrm{~h}^{-1} \mathrm{~m}^{-1}$; no segundo ano, registrou a remoção média no Pt de $71 \%$ para a

Tabela 2. Valores médios de concentração e de remoção de fósforo do esgoto, em diferentes distâncias percorridas em rampas de tratamento por escoamento superficial e para diferentes taxas de aplicação

\begin{tabular}{|c|c|c|c|c|c|c|c|c|}
\hline \multirow{2}{*}{ Variável } & \multirow{2}{*}{$\begin{array}{l}\text { Taxa de Aplicação } \\
\qquad\left(\mathrm{m}^{3} \mathrm{~h}^{-1} \mathrm{~m}^{-1}\right)\end{array}$} & & \multicolumn{6}{|c|}{ Distância (m) } \\
\hline & & & 0 & 2 & 4 & 6 & 8 & 10 \\
\hline \multirow{6}{*}{ Pt } & \multirow{2}{*}{0,24} & Concentração (mg L-1) & 9,80 & 8,90 & 8,33 & 7,05 & 5,15 & - \\
\hline & & Remoção (\%) & 0,00 & 9,15 & 14,99 & 28,04 & 47,46 & - \\
\hline & \multirow{2}{*}{0,36} & Concentração (mg L-1) & 9,63 & 10,02 & 8,98 & 8,90 & 7,86 & 5,73 \\
\hline & & Remoção (\%) & 0,00 & $-4,06$ & 6,77 & 7,57 & 18,37 & 40,47 \\
\hline & \multirow{2}{*}{0,48} & Concentração (mg L-1) & 9,75 & 10,43 & 9,53 & 8,90 & 9,50 & 8,71 \\
\hline & & Remoção (\%) & 0,00 & $-6,98$ & 2,19 & 8,69 & 2,48 & 10,63 \\
\hline \multirow{6}{*}{ Ptr } & \multirow{2}{*}{0,24} & Concentração (mg L-1) & 2,58 & 2,37 & 2,05 & 1,91 & 1,36 & - \\
\hline & & Remoção (\%) & 0,00 & 8,06 & 20,60 & 26,20 & 47,50 & - \\
\hline & \multirow{2}{*}{0,36} & Concentração (mg L-1) & 2,52 & 3,25 & 2,22 & 2,28 & 1,12 & 1,10 \\
\hline & & Remoção (\%) & 0,00 & $-29,07$ & 11,81 & 9,40 & 55,65 & 56,10 \\
\hline & \multirow{2}{*}{0,48} & Concentração (mg L-1) & 2,41 & 3,25 & 2,68 & 2,18 & 2,96 & 2,22 \\
\hline & & Remoção (\%) & 0,00 & $-35,14$ & $-11,24$ & 9,61 & $-22,85$ & 7,81 \\
\hline \multirow{6}{*}{ Ptd } & \multirow{2}{*}{0,24} & Concentração (mg L-1) & 7,22 & 6,53 & 6,28 & 5,15 & 3,79 & - \\
\hline & & Remoção (\%) & 0,00 & 9,54 & 12,98 & 28,70 & 47,45 & - \\
\hline & \multirow{2}{*}{0,36} & Concentração (mg L-1) & 7,12 & 6,78 & 6,76 & 6,62 & 6,75 & 4,63 \\
\hline & & Remoção (\%) & 0,00 & 4,78 & 4,98 & 6,93 & 5,19 & 34,94 \\
\hline & \multirow{2}{*}{0,48} & Concentração (mg L-1) & 7,34 & 7,17 & 6,85 & 6,72 & 6,55 & 6,49 \\
\hline & & Remoção (\%) & 0,00 & 2,16 & 6,60 & 8,39 & 10,79 & 11,56 \\
\hline \multirow{6}{*}{ Pid } & \multirow{2}{*}{0,24} & Concentração (mg L-1) & 6,65 & 6,03 & 6,36 & 4,84 & 3,82 & - \\
\hline & & Remoção (\%) & 0,00 & 9,32 & 4,39 & 27,23 & 42,60 & - \\
\hline & \multirow{2}{*}{0,36} & Concentração (mg L-1) & 7,22 & 7,05 & 6,93 & 6,38 & 6,66 & 4,60 \\
\hline & & Remoção (\%) & 0,00 & 2,25 & 3,96 & 11,58 & 7,77 & 36,28 \\
\hline & \multirow{2}{*}{0,48} & Concentração (mg L-1) & 7,04 & 6,98 & 6,95 & 6,42 & 6,32 & 6,20 \\
\hline & & Remoção (\%) & 0,00 & 0,98 & 1,35 & 8,91 & 10,21 & 11,94 \\
\hline \multirow{6}{*}{ Pod } & \multirow{2}{*}{0,24} & Concentração (mg L-1) & 0,91 & 0,76 & 0,32 & 0,53 & 0,13 & - \\
\hline & & Remoção (\%) & 0,00 & 16,70 & 64,18 & 41,59 & 85,12 & - \\
\hline & \multirow{2}{*}{0,36} & Concentração (mg L-1) & 0,95 & 0,73 & 0,65 & 0,45 & 0,42 & 0,03 \\
\hline & & Remoção (\%) & 0,00 & 23,45 & 31,00 & 52,30 & 55,59 & 96,62 \\
\hline & \multirow{2}{*}{0,48} & Concentração (mg L-1) & 0,61 & 0,85 & 0,69 & 0,64 & 0,35 & 0,48 \\
\hline & & Remoção (\%) & 0,00 & $-39,27$ & $-12,78$ & $-5,04$ & 43,53 & 20,88 \\
\hline
\end{tabular}

Pt - Fósforo total; Ptr - Fósforo total no resíduo; Ptd - Fósforo total dissolvido; Pid - Fósforo inorgânico dissolvido; Pod - Fósforo orgânico dissolvido 
taxa $0,15 \mathrm{~m}^{3} \mathrm{~h}^{-1} \mathrm{~m}^{-1}$ e de $50 \%$ para a taxa $0,30 \mathrm{~m}^{3} \mathrm{~h}^{-1} \mathrm{~m}^{-1}$; enquanto no terceiro ano, a remoção média de Pt ficou em valores próximos a $50 \%$ para todas as taxas aplicadas.

Taxas de aplicação menores proporcionam maior tempo de contato entre a água residuária, o solo e o filme biológico, formado na superfície do solo e no colo das plantas. Taxas menores devem ser, por esta razão, recomendadas para aplicação de esgoto doméstico submetido a tratamento preliminar apenas em sistemas de tratamento por escoamento superficial.

Taxas de aplicação maiores proporcionam menor tempo de detenção do esgoto no solo e no filme biológico. Outro fator é que essas taxas implicam níveis de saturação do solo mais elevados e a umidade excessiva nas camadas mais superficiais limita e desequilibra a atividade biológica, por dificultar a aeração do solo (Dommergues \& Mangenot, 1970).

As remoções elevadas de Pod (até 85,12\%) verificadas nas rampas de tratamento que receberam taxas de aplicação de $0,24 \mathrm{~m}^{3} \mathrm{~h}^{-1} \mathrm{~m}^{-1}$ dão indícios de que esta taxa seja, dentre as avaliadas, a que proporcionou carga orgânica mais próxima da capacidade do sistema em degradar o material orgânico dissolvido.
A Tabela 3 apresenta os modelos linear e exponencial, respectivamente, para as variáveis Pt, Ptr, Ptd, Pid e Pod, para o estádio 1 de remoção de fósforo, tendo em vista a não-disponibilidade de dados a partir dos $10 \mathrm{~m}$ de comprimento das rampas de escoamento superficial.

Verificaram-se pequenas discrepâncias entre os valores de $\mathrm{r}^{2}$, no modelo linear, e $\mathrm{R}^{2}$, no modelo exponencial, Tabela 3. Considerando-se que o modelo mais comum para a remoção de fósforo em sistemas de tratamento de águas residuárias por escoamento superficial é o exponencial, este deve ser recomendado, por manter a coerência com aqueles encontrados na literatura (Abernathy et al., 1985).

Os coeficientes para os modelos de remoção de fósforo no estádio 1, Tabela 4, e os encontrados por Abernathy et al. (1985), embora obtidos para diferentes comprimento e declividade da rampa de escoamento, variados tipos de água residuária aplicada e diferentes taxas de aplicação, foram comparados entre si, Tabela 5. Os coeficientes K e K’ foram calculados utilizando-se as equações 3 e 4, respectivamente.

Comparando-se os coeficientes do modelo exponencial $\mathrm{k}=0,00194$ e $\mathrm{n}=2,31368$, obtidos neste experimento para as taxas de aplicação de efluente primário 0,24 a $0,48 \mathrm{~m}^{3} \mathrm{~h}^{-1} \mathrm{~m}^{-1}$,

Tabela 3. Equações lineares e exponenciais ajustadas para fósforo, em função da distância para as três taxas de aplicação, com os respectivos coeficientes de determinação e remoções simuladas após $8 \mathrm{~m}$ de escoamento na rampa

\begin{tabular}{|c|c|c|c|c|c|c|c|}
\hline Variável & $\begin{array}{c}\text { Taxa de } \\
\text { Aplicação } \\
\left(\mathrm{m}^{3} \mathrm{~h}^{-1} \mathrm{~m}^{-1}\right)\end{array}$ & $\begin{array}{l}\text { Modelo Linear } \\
\quad(x \text { em } \mathrm{m})\end{array}$ & $\mathrm{r}^{2}$ & $\begin{array}{c}\text { Remoção } \\
\text { Simulada } \\
(\%)\end{array}$ & $\begin{array}{l}\text { Modelo Exponencial } \\
(x \text { em } \mathrm{m})\end{array}$ & $\mathrm{R}^{2}$ & $\begin{array}{c}\text { Remoção } \\
\text { Simulada } \\
(\%)\end{array}$ \\
\hline \multirow{3}{*}{ Pt } & 0,24 & $\frac{\mathrm{C}}{\mathrm{C}_{0}}=1,004-0,045 \mathrm{x}$ & 0,9862 & 36,02 & $\frac{C}{C_{0}}=1,008 \exp (-0,051 x)$ & 0,9717 & 33,93 \\
\hline & 0,36 & $\frac{\mathrm{C}}{\mathrm{C}_{0}}=1,029-0,021 \mathrm{x}$ & 0,7770 & 17,00 & $\frac{C}{C_{0}}=1,029 \exp (-0,022 x)$ & 0,7616 & 16,65 \\
\hline & 0,48 & $\frac{\mathrm{C}}{\mathrm{C}_{0}}=1,023-0,010 \mathrm{x}$ & 0,3359 & 7,97 & $\frac{C}{C_{0}}=1,023 \exp (-0,010 \mathrm{x})$ & 0,3369 & 8,03 \\
\hline \multirow{3}{*}{ Ptr } & 0,24 & $\frac{\mathrm{C}}{\mathrm{C}_{0}}=0,979-0,042 \mathrm{x}$ & 0,8809 & 33,89 & $\frac{\mathrm{C}}{\mathrm{C}_{0}}=0,985 \exp (-0,051 \mathrm{x})$ & 0,8988 & 33,05 \\
\hline & 0,36 & $\frac{C}{C_{0}}=0,999-0,037 x$ & 0,6701 & 29,93 & $\frac{C}{C_{0}}=1,009 \exp (-0,046 x)$ & 0,6994 & 31,08 \\
\hline & 0,48 & $\frac{\mathrm{C}}{\mathrm{C}_{0}}=1,020-0,023 \mathrm{x}$ & 0,4289 & 18,66 & $\frac{C}{C_{0}}=1,024 \exp (-0,026 x)$ & 0,4363 & 19,10 \\
\hline \multirow{3}{*}{ Ptd } & 0,24 & $\frac{\mathrm{C}}{\mathrm{C}_{0}}=1,011-0,046 \mathrm{x}$ & 0,8979 & 36,37 & $\frac{C}{C_{0}}=1,013 \exp (-0,051 \mathrm{x})$ & 0,8729 & 33,81 \\
\hline & 0,36 & $\frac{C}{C_{0}}=0,995-0,013 x$ & 0,5631 & 10,21 & $\frac{C}{C_{0}}=0,995 \exp (-0,013 \mathrm{x})$ & 0,5564 & 10,09 \\
\hline & 0,48 & $\frac{\mathrm{C}}{\mathrm{C}_{0}}=0,987-0,010 \mathrm{x}$ & 0,7464 & 7,78 & $\frac{C}{C_{0}}=0,988 \exp (-0,011 \mathrm{x})$ & 0,7576 & 7,94 \\
\hline \multirow{3}{*}{ Pid } & 0,24 & $\frac{C}{C_{0}}=1,005-0,040 \mathrm{x}$ & 0,7883 & 31,87 & $\frac{C}{C_{0}}=1,006 \exp (-0,044 x)$ & 0,7673 & 29,84 \\
\hline & 0,36 & $\frac{\mathrm{C}}{\mathrm{C}_{0}}=1,011-0,020 \mathrm{x}$ & 0,7839 & 15,71 & $\frac{C}{C_{0}}=1,012 \exp (-0,021 x)$ & 0,7797 & 15,62 \\
\hline & 0,48 & $\frac{C}{C_{0}}=1,016-0,010 x$ & 0,7356 & 7,98 & $\frac{C}{C_{0}}=1,016 \exp (-0,010 x)$ & 0,7301 & 7,96 \\
\hline \multirow{3}{*}{ Pod } & 0,24 & $\frac{C}{C_{0}}=0,947-0,088 x$ & 0,8382 & 70,17 & $\frac{C}{C_{0}}=0,982 \exp (-0,145 x)$ & 0,9262 & 67,28 \\
\hline & 0,36 & $\frac{\mathrm{C}}{\mathrm{C}_{0}}=0,995-0,063 \mathrm{x}$ & 0,9815 & 50,68 & $\frac{C}{C_{0}}=1,003 \exp (-0,081 x)$ & 0,9698 & 47,88 \\
\hline & 0,48 & $\frac{\mathrm{C}}{\mathrm{C}_{0}}=0,982-0,029 \mathrm{x}$ & 0,3553 & 23,55 & $\frac{C}{C_{0}}=0,991 \exp (-0,036 x)$ & 0,3768 & 24,99 \\
\hline
\end{tabular}

Pt - Fósforo total; Ptr - Fósforo total no resíduo; Ptd - Fósforo total dissolvido; Pid - Fósforo inorgânico dissolvido; Pod - Fósforo orgânico dissolvido 
Tabela 4. Coeficientes dos modelos de remoção de fósforo no estádio 1 e respectivos coeficientes de correlação

\begin{tabular}{|c|c|c|c|c|}
\hline \multirow{2}{*}{ Variável } & \multirow{2}{*}{ Modelo } & \multicolumn{2}{|c|}{ Coeficientes } & \multirow{2}{*}{$\mathbf{r}$} \\
\hline & & $\mathbf{k}$ & n & \\
\hline \multirow{2}{*}{ Pt } & Linear & 0,02127 & 0,14611 & 0,9795 \\
\hline & Exponencial & 0,00194 & 2,31368 & 0,9975 \\
\hline \multirow{2}{*}{ Ptr } & Linear & 0,03144 & 0,07930 & 0,9636 \\
\hline & Exponencial & 0,01470 & 0,93652 & 0,8873 \\
\hline \multirow{2}{*}{ Ptd } & Linear & 0,01881 & 0,14890 & 0,9017 \\
\hline & Exponencial & 0,00163 & 2,34421 & 0,9607 \\
\hline \multirow{2}{*}{ Pid } & Linear & 0,01992 & 0,12442 & 0,9799 \\
\hline & Exponencial & 0,00230 & 2,08953 & 0,9960 \\
\hline \multirow{2}{*}{ Pod } & Linear & 0,04536 & 0,24282 & 0,9956 \\
\hline & Exponencial & 0,00952 & 1,95454 & 0,9815 \\
\hline
\end{tabular}

dissolvido; Pod - Fósforo orgânico dissolvido
disto

Tabela 5. Coeficientes $\mathrm{k}$ e $\mathrm{n}$ sugeridos para o modelo exponencial de remoção de fósforo total, com respectivos coeficientes $\mathrm{K}$ calculados, para diferentes estádios, tipos de efluente aplicado, declividades da rampa de escoamento e taxas de aplicação

\begin{tabular}{|c|c|c|c|c|c|c|c|}
\hline \multirow{2}{*}{ Fonte } & \multirow{2}{*}{ Estádio } & \multirow{2}{*}{$\begin{array}{c}\text { Tipo de Efluente } \\
\text { Aplicado }\end{array}$} & \multirow{2}{*}{$\begin{array}{c}\text { Declividade } \\
(\%)\end{array}$} & \multirow{2}{*}{$\begin{array}{l}\text { Taxa de Aplicação } \\
\left(\mathrm{m}^{3} \mathrm{~h}^{-1} \mathrm{~m}^{-1}\right)\end{array}$} & \multicolumn{2}{|c|}{ Coeficientes } & \multirow{2}{*}{$\begin{array}{c}\text { Equação } 3 \text { ou } 4 \\
\text { K ou K' }\end{array}$} \\
\hline & & & & & k ou k' & n ou n' & \\
\hline Tese $^{1}$ & 1 & Preliminar & 2 & 0,24 a 0,48 & 0,00194 & 2,31368 & 0,05281 \\
\hline Abernathy et al. & 2 & Bruto & 4 & 0,21 a 0,45 & 0,01217 & 0,17521 & 0,01563 \\
\hline
\end{tabular}

${ }^{1}$ Dados obtidos no presente trabalho

em rampas de escoamento com declividade de $2 \%$, com os valores k' $=0,01217$ e n' $=0,17521$ sugeridos por Abernathy et al. (1985), para as taxas de aplicação de efluente bruto 0,21 a $0,45 \mathrm{~m}^{3} \mathrm{~h}^{-1} \mathrm{~m}^{-1}$, em rampas com declividade de $4 \%$, verifica-se que os mesmos são diferentes, gerando variados valores para $\mathrm{K}$ e K', respectivamente. Maiores coeficientes K e K' proporcionam menores relações $\mathrm{C}_{\mathrm{x}} / \mathrm{C}_{0}$; portanto, maiores remoções; assim, o coeficiente $K=0,05281$, obtido neste experimento, deverá proporcionar maior remoção de $\mathrm{P}$ do esgoto doméstico que o K’ = 0,01563 sugerido por Abernathy et al. (1985).

\section{Alteração na concentração de fósforo no solo no início das rampas de tratamento}

As menores concentrações de fósforo nas amostras de solo coletadas na área testemunha se devem ao fato de que as áreas compreendidas pelas rampas de aplicação de esgoto doméstico já haviam sido utilizadas em experimento anterior, com aplicação da mesma água residuária, Tabela 6. A área testemunha não recebeu nenhum esgoto previamente.

Para a taxa de aplicação $0,24 \mathrm{~m}^{3} \mathrm{~h}^{-1} \mathrm{~m}^{-1}$, na camada $\mathrm{C}_{1}$, ocorreu diminuição no conteúdo de P-disponível, de 33,75 para 26,70 $\mathrm{mg} \mathrm{dm}^{-3}$; para a taxa $0,48 \mathrm{~m}^{3} \mathrm{~h}^{-1} \mathrm{~m}^{-1}$, de 49,70 para $15,10 \mathrm{mg} \mathrm{dm}^{-3} \mathrm{e}$, para a testemunha, que não recebeu esgoto, de 2,30 para $0,90 \mathrm{mg} \mathrm{dm}^{-3}$, correspondendo a diminuições de 21 , 70 e $61 \%$, respectivamente. Na camada $C_{1}$, Fonseca (2000), trabalhando com a taxa $0,24 \mathrm{~m}^{3} \mathrm{~h}^{-1} \mathrm{~m}^{-1}$, registrou aumento no conteúdo de fósforo de 9,6 para $14,4 \mathrm{mg} \mathrm{dm}^{-3}$, referente a aumento de $33 \%$. Na camada $C_{2}$, para a taxa de aplicação $0,24 \mathrm{~m}^{3} \mathrm{~h}^{-1} \mathrm{~m}^{-1}$, houve diminuição no conteúdo de P-disponível, de 8,70 para 3,25 $\mathrm{mg} \mathrm{dm}^{-3}$; para a taxa $0,48 \mathrm{~m}^{3} \mathrm{~h}^{-1} \mathrm{~m}^{-1}$, de 5,40 para $2,30 \mathrm{mg} \mathrm{dm}^{-3}$ e, para a testemunha, de 2,50 para 0,25 $\mathrm{mg} \mathrm{dm}^{-3}$, concernente a diminuições de 63, 57 e $90 \%$, respectivamente. Na camada $C_{2}$, esse mesmo autor, trabalhando com a taxa $0,24 \mathrm{~m}^{3} \mathrm{~h}^{-1} \mathrm{~m}^{-1}$, também registrou diminuição no conteúdo de fósforo, de 8,7 para $6,6 \mathrm{mg} \mathrm{dm}^{-3}$, correspondendo à diminuição de $24 \%$, Tabela 6.

No caso da taxa de aplicação $0,24 \mathrm{~m}^{3} \mathrm{~h}^{-1} \mathrm{~m}^{-1}$, suspeitase que o conteúdo de fósforo adicionado ao solo tenha sido suficientemente baixo para haver grande competição (plantas, solo, microrganismos) por este elemento, diminuindo sua disponibilidade no meio. No caso da taxa de aplicação $0,48 \mathrm{~m}^{3} \mathrm{~h}^{-1} \mathrm{~m}^{-1}$, acredita-se que o grande aporte de material orgânico, favorecendo a quelação/complexação do Pdisponível, possa ser a principal razão para explicar a diminuição de sua disponibilidade. A menor disponibilidade

Tabela 6. Alteração na concentração de fósforo disponível, matéria orgânica, pH, acidez, soma de bases trocáveis e capacidade de troca catiônica, nas camadas de 0 a $10 \mathrm{~cm}$ e de 50 a $60 \mathrm{~cm}$, antes e depois da aplicação da água residuária, no solo do início das rampas de tratamento (1 m), para diferentes taxas de aplicação

\begin{tabular}{|c|c|c|c|c|c|c|c|c|c|c|c|c|c|}
\hline \multirow{3}{*}{$\begin{array}{c}\text { Taxa de } \\
\text { aplicação } \\
\left(\mathrm{m}^{3} \mathrm{~h}^{-1} \mathrm{~m}^{-1}\right)\end{array}$} & & \multirow{2}{*}{\multicolumn{2}{|c|}{$\begin{array}{c}P \\
\left(\mathrm{mg} \mathrm{dm}^{-3}\right)\end{array}$}} & \multirow{2}{*}{\multicolumn{2}{|c|}{$\begin{array}{c}\text { MO } \\
\left(\text { dag }^{-1} \mathrm{~kg}^{-1}\right)\end{array}$}} & \multirow{2}{*}{\multicolumn{2}{|c|}{$\mathrm{pH}\left(\mathrm{H}_{2} \mathrm{O}\right)$}} & \multicolumn{2}{|c|}{$\mathrm{H}+\mathrm{Al}$} & \multirow{2}{*}{\multicolumn{2}{|c|}{$\begin{array}{c}\text { SB } \\
\left(\mathrm{cmol}_{\mathrm{c}} \mathrm{dm}^{-3}\right)\end{array}$}} & \multicolumn{2}{|c|}{$T$} \\
\hline & & & & & & & & & & & & & \\
\hline & & $\mathrm{C}_{1}$ & $\mathrm{C}_{2}$ & $c_{1}$ & $\mathrm{C}_{2}$ & $c_{1}$ & $\mathrm{C}_{2}$ & $C_{1}$ & $\mathrm{C}_{2}$ & $C_{1}$ & $\mathrm{C}_{2}$ & $C_{1}$ & $\mathrm{C}_{2}$ \\
\hline \multirow{2}{*}{0,24} & Antes & 33,75 & 8,70 & 5,24 & 1,86 & 5,60 & 5,35 & 4,15 & 2,80 & 2,62 & 1,53 & 6,77 & 4,33 \\
\hline & Depois & 26,70 & 3,25 & 2,26 & 2,04 & 5,72 & 5,46 & 3,70 & 2,15 & 4,92 & 2,18 & 8,62 & 4,33 \\
\hline \multirow{2}{*}{0,36} & Antes & 28,70 & 1,70 & 4,74 & 1,45 & 5,60 & 5,45 & 3,30 & 1,85 & 2,46 & 1,04 & 5,76 & 2,89 \\
\hline & Depois & 31,10 & 4,15 & 2,64 & 1,32 & 5,87 & 5,42 & 3,40 & 2,50 & 3,28 & 1,57 & 6,68 & 4,07 \\
\hline \multirow{2}{*}{0,48} & Antes & 49,70 & 5,40 & 5,21 & 1,51 & 5,60 & 5,50 & 4,30 & 2,00 & 3,35 & 1,12 & 7,65 & 3,12 \\
\hline & Depois & 15,10 & 2,30 & 2,26 & 2,39 & 5,30 & 5,49 & 3,20 & 1,80 & 2,29 & 5,00 & 5,49 & 6,80 \\
\hline \multirow{2}{*}{ Testemunha } & Antes & 2,30 & 2,50 & 2,58 & 1,04 & 5,50 & 5,35 & 3,45 & 1,15 & 2,05 & 0,79 & 5,50 & 1,94 \\
\hline & Depois & 0,90 & 0,25 & 2,23 & 2,07 & 5,55 & 5,40 & 3,05 & 1,85 & 3,81 & 1,80 & 6,86 & 3,65 \\
\hline
\end{tabular}


de fósforo no solo pode, também, estar associada à sua imobilização junto aos oxidróxidos de ferro e alumínio e à biomassa microbiana.

Para a taxa de aplicação $0,36 \mathrm{~m}^{3} \mathrm{~h}^{-1} \mathrm{~m}^{-1}$, constatou-se aumento no conteúdo de fósforo, de 28,70 para $31,10 \mathrm{mg} \mathrm{dm}^{-3}$, na camada $\mathrm{C}_{1}$, e de 1,70 para $4,15 \mathrm{mg} \mathrm{dm}^{-3}$, na camada $\mathrm{C}_{2}$, referente a aumentos de 8 e 59\%, respectivamente. Fonseca (2000), trabalhando com a mesma taxa de aplicação, também registrou aumentos no conteúdo de fósforo, de 8,8 para 22,6 $\mathrm{mg} \mathrm{dm}^{-3}$, na camada $C_{1}$, e de 1,8 para $9,3 \mathrm{mg} \mathrm{dm}^{-3}$, na camada $C_{2}$, representando aumentos de 61 e $81 \%$, respectivamente.

O solo da área experimental apresentou, após o experimento, tendência de aumento nas cargas negativas, como evidenciado pelo aumento de $\mathrm{T}$, provavelmente associado ao aumento do $\mathrm{pH}$, uma vez que o conteúdo de MO, material sabidamente de alta CTC, diminuiu na camada mais superficial do solo (0 a $10 \mathrm{~cm}$ ). Solos com predominância de caulinita e oxidróxidos de ferro e alumínio na fração argila, têm carga $\mathrm{pH}$-dependente, que aumenta com a elevação do $\mathrm{pH}$.

O aumento de T na rampa testemunha, por sua vez, pode estar associado à maior humificação do material orgânico presente proporcionando, assim, aumento na quantidade de cargas negativas no meio.

\section{Remoção do fósforo pela planta}

A maior remoção de fósforo pela planta para a taxa de aplicação $0,36 \mathrm{~m}^{3} \mathrm{~h}^{-1} \mathrm{~m}^{-1}$ pode estar relacionada ao aumento no conteúdo de P-disponível no solo, em ambas as camadas avaliadas, para a referida taxa, Tabela 7.

Haddad \& Castro (1998) apresentaram uma revisão de trabalhos envolvendo espécies forrageiras passíveis de serem

Tabela 7. Número de cortes, data, idade, concentração de fósforo e remoção do elemento pelo capim-coastcross, em relação à matéria seca (MS), para diferentes taxas de aplicação

\begin{tabular}{|c|c|c|c|c|c|}
\hline $\begin{array}{c}\text { Taxa de } \\
\text { Aplicação } \\
\left(\mathrm{m}^{3} \mathrm{~h}^{-1} \mathrm{~m}^{-1}\right)\end{array}$ & Corte & Data & $\begin{array}{l}\text { Idade } \\
\text { (dias) }\end{array}$ & $\begin{array}{c}P \\
\left(g^{~ k g-1}\right)\end{array}$ & $\begin{array}{c}\text { Remoção } \\
\left(\mathrm{kg} \mathrm{ha}^{-1} \text { de P) }\right.\end{array}$ \\
\hline \multirow{5}{*}{0,24} & 1 & 09/08/01 & 45 & 4,90 & 2,38 \\
\hline & 2 & $22 / 09 / 01$ & 44 & 4,50 & 1,91 \\
\hline & 3 & 09/11/01 & 48 & 4,50 & 7,80 \\
\hline & Média & & - & 4,63 & - \\
\hline & Total & & - & - & 12,09 \\
\hline \multirow{5}{*}{0,36} & 1 & 09/08/01 & 45 & 5,22 & 5,19 \\
\hline & 2 & $22 / 09 / 01$ & 44 & 5,07 & 5,74 \\
\hline & 3 & $09 / 11 / 01$ & 48 & 3,59 & 4,85 \\
\hline & Média & & - & 4,63 & - \\
\hline & Total & & - & - & 15,78 \\
\hline \multirow{5}{*}{0,48} & 1 & 09/08/01 & 45 & 4,94 & 2,62 \\
\hline & 2 & $22 / 09 / 01$ & 44 & 4,27 & 3,45 \\
\hline & 3 & $09 / 11 / 01$ & 48 & 3,94 & 2,30 \\
\hline & Média & & - & 4,38 & - \\
\hline & Total & & - & - & 8,37 \\
\hline \multirow{5}{*}{ Testemunha } & 1 & 09/08/01 & 45 & 2,26 & 0,94 \\
\hline & 2 & $22 / 09 / 01$ & 44 & 2,41 & 1,02 \\
\hline & 3 & $09 / 11 / 01$ & 48 & 2,29 & 1,20 \\
\hline & Média & & - & 2,32 & - \\
\hline & Total & & - & - & 3,16 \\
\hline
\end{tabular}

fenadas e suas características nutricionais em função da idade de corte. Para o capim-coastcross, foi encontrado um conteúdo de fósforo de $2 \mathrm{~g} \mathrm{~kg}^{-1}$, nos cortes efetuados aos 40 e 50 dias de idade; este valor é 54\% inferior à concentração média mais baixa obtida neste trabalho, correspondendo a $4,38 \mathrm{~g} \mathrm{~kg}^{-1}$, sob a taxa de aplicação $0,48 \mathrm{~m}^{3} \mathrm{~h}^{-1} \mathrm{~m}^{-1}$.

Fonseca (2000), em estudo conduzido na mesma área com o capim-coastcross e aplicação de esgoto doméstico, verificou concentrações médias de fósforo de $3,7 \mathrm{~g} \mathrm{~kg}^{-1}$, sob a taxa de aplicação $0,24 \mathrm{~m}^{3} \mathrm{~h}^{-1} \mathrm{~m}^{-1}$; de $3,8 \mathrm{~g} \mathrm{~kg}^{-1}$, sob a taxa $0,36 \mathrm{~m}^{3} \mathrm{~h}^{-1} \mathrm{~m}^{-1}$ e de $2,2 \mathrm{~g} \mathrm{~kg}^{-1}$ para a testemunha, sendo 20 , 19 e apenas $5 \%$, respectivamente, inferiores aos valores encontrados neste experimento.

Queiroz (2000), trabalhando com o capim-coastcross, notou concentrações médias de fósforo de $4,0 \mathrm{~g} \mathrm{~kg}^{-1}$, sob aplicação de água residuária de suinocultura, e de 4,4 g kg-1, sob água da rede de abastecimento.

Observou-se um ponto teórico de máximo, correspondendo à taxa de aplicação $0,34 \mathrm{~m}^{3} \mathrm{~h}^{-1} \mathrm{~m}^{-1}$, quando a concentração estimada de fósforo na MS do capim-coastcross é máxima, atingindo o valor de $4,75 \mathrm{~g} \mathrm{~kg}^{-1}$.

\section{CONCLUSÕES}

1. Na avaliação do tratamento de esgoto doméstico em sistemas por escoamento superficial, em até $8 \mathrm{~m}$ de rampa, verificou-se que a menor taxa de aplicação, $0,24 \mathrm{~m}^{3} \mathrm{~h}^{-1} \mathrm{~m}^{-1}$, resultou na maior eficiência na remoção de fósforo, tendo alcançado eficiências de 47,46\% para Pt; 47,45\% para Ptd; 42,60\% para Pid e 85,12\% para Pod; no entanto, para Ptr, a taxa de aplicação $0,36 \mathrm{~m}^{3} \mathrm{~h}^{-1} \mathrm{~m}^{-1}$ resultou em maior eficiência de remoção de fósforo, com o valor de 55,65\%.

2. Os coeficientes para o estágio 1 do modelo exponencial foram $\mathrm{k}=0,0019$ e $\mathrm{n}=2,3137$ para Pt, $\mathrm{k}=0,0147$ e $\mathrm{n}=0,9365$ para Ptr, $\mathrm{k}=0,0016$ e $\mathrm{n}=2,3442$ para Ptd, $\mathrm{k}=0,0023$ e $\mathrm{n}=2,0895$ para Pid, e $\mathrm{k}=0,0095$ e $\mathrm{n}=1,9545$ para Pod.

3. Na avaliação do solo em relação ao início do experimento, para as taxas de aplicação 0,24 e $0,48 \mathrm{~m}^{3} \mathrm{~h}^{-1} \mathrm{~m}^{-1}$ e para a testemunha, houve diminuições no conteúdo de P-disponível, correspondendo a 21, 70 e 61\%, respectivamente, na camada de 0 a $10 \mathrm{~cm}$, e 63, 57 e 90\%, respectivamente, na camada de 50 a $60 \mathrm{~cm}$, ambas a $1 \mathrm{~m}$ do início das rampas de tratamento. Para a taxa de aplicação $0,36 \mathrm{~m}^{3} \mathrm{~h}^{-1} \mathrm{~m}^{-1}$, ocorreu aumento no conteúdo de P-disponível, correspondendo a 8\%, na camada de 0 a $10 \mathrm{~cm}$, e $59 \%$, na camada de 50 a $60 \mathrm{~cm}$.

4. Na avaliação da forrageira, a taxa de aplicação $0,36 \mathrm{~m}^{3} \mathrm{~h}^{-1} \mathrm{~m}^{-1}$ apresentou maior remoção de fósforo pela planta, $15,78 \mathrm{~kg} \mathrm{ha}^{-1}$ de $\mathrm{P}$.

\section{AgRADECIMENTOS}

Ao Departamento de Engenharia Agrícola (DEA) da Universidade Federal de Viçosa (UFV), à Companhia de Saneamento do Estado de Minas Gerais (COPASA) e à Fundação de Amparo à Pesquisa do Estado de Minas Gerias (FAPEMIG) pelo auxílio à pesquisa e suporte financeiro. 


\section{LITERATURA CITADA}

Abernathy, A. R.; Zirschky, J.; Borup, M. B. Overland flow wastewater treatment at Easley, SC. Journal of Water Pollution Control Federation (JWPCF), Washington, v.57, n.4, p.291-299, 1985.

Alvarez Venegas, V. H.; Dias, L. E.; Ribeiro Jr.; E. S.; Souza, R. B. de; Fonseca, C. A. da. Métodos de análises de enxofre em solos e plantas. Viçosa, MG: UFV, 1999. 131p.

APHA - American Public Health Association, AWWA - American Water Works Association, WEF - Water Environment Federation. Standard methods for the examination of water and wastewater. 18 $8^{\text {th }}$ ed. Washington: APHA, 1992.

Black, S. A. Experience with phosphorus removal at existing Ontario municipal wastewater treatment plants. In: Loehr, R.C.; Martin, C.S.; Rast, W. (eds). Phosphorus management strategies for lakes. Ann Arbor, Michigan: Ann Arbor Science Publishers, Inc., 1980. chap.13, p.329-353.

Brasil. Resolução CONAMA nº 357, de 17 de março de 2005. Diário Oficial da União, Brasília, DF, 18 mar. 2005, 24p. Disponível em: http://www.mma.gov.br/port/conama/res/ res05/res35705.pdf. Acesso em: 12 maio 2005.

Campos, J. R. (Coor.). Tratamento de esgotos sanitários por processo anaeróbio e disposição controlada no solo. Rio de Janeiro: Associação Brasileira de Engenharia Sanitária e Ambiental (ABES), 1999. 435p.

Coraucci Filho, B. Tratamento de esgotos domésticos no solo pelo método do escoamento superficial. São Paulo: USP, 1991. 1336p. Tese Doutorado

Dommergues, Y.; Mangenot, F. Écologie microbienne du sol. Paris: Masson et Cie, Éditeurs, 1970. 796p.

EMBRAPA - Empresa Brasileira de Pesquisa Agropecuária. Manual de métodos de análise do solo, $2^{\mathrm{a}}$ ed. Rio de Janeiro: Centro Nacional de Pesquisa de Solos, 1997. 212p.
EMBRAPA - Empresa Brasileira de Pesquisa Agropecuária. Sistema brasileiro de classificação de solos. Brasília: Embrapa Produção de Informação; Rio de Janeiro: Embrapa Solos, 1999. 412p.

Esteves, F. A. Fundamentos de limnologia. Rio de Janeiro: Editora Interciência Ltda., 1988. 575p.

Fonseca, S. P. P. Tratamento de esgoto doméstico bruto pelo método de escoamento superficial utilizando o capim coastcross (Cynodon dactylon (L.) Pers.). Viçosa, MG: UFV, 2000. 133p. Dissertação Mestrado.

Haddad, C. M.; Castro, F. G. F. Produção de feno. In: Simpósio sobre Manejo da Pastagem, 15, 1998, Piracicaba. Anais... Piracicaba: FEALQ, 1998. p.151.

Metcalf \& Eddy, Inc. Wastewater engineering: treatment and reuse. Revised by George Tchobanoglous, Franklin L. Burton, H. David Stensel, $4^{\text {th }}$ ed. New York: McGraw-Hill Publishing Company, 2003. 1819p.

Novais, R. F.; Smyth, T. J. Fósforo em solo e planta em condições tropicais. Viçosa, MG: UFV/DPS, 1999. 399p.

Paganini, W. S. Disposição de esgotos no solo. $2^{\mathrm{a}}$ ed. São Paulo: Fundo Editorial da AESABESP, 1997. 232p.

Queiroz, F. M. Avaliação de gramíneas forrageiras para o tratamento de águas residuárias da suinocultura. Viçosa, MG: UFV, 2000. 91p. Dissertação Mestrado.

Silva, D. J.; Queiroz, A. C. de. Análise de alimentos: métodos químicos e biológicos. $3^{\mathrm{a}}$ ed. Viçosa, MG: UFV, 2002. 235p.

Silva, F. C. (Org.). Manual de análises químicas de solos, plantas e fertilizantes. Brasília: EMBRAPA Comunicação para Transferência de Tecnologia, 1999. 370p.

Smith, R. G.; Schroeder, E. D. Field studies of the overland flow process for the treatment of raw and primary treated municipal wastewater. Journal of Water Pollution Control Federation (JWPCF), Washington, v.57, n.7, p.785-794, 1985.

USEPA - United States Environmental Protection Agency; U.S. Army Corps of Engineers; U.S. Department of Interior; U.S. Department of Agriculture. Process design manual for land treatment of municipal wastewater. Cincinnati: EPA, 1981. 